\title{
Interfacial Behaviour in Tunnel Engineering
}

\author{
SEAN BohUn ${ }^{1} \dagger$ \\ 1 Ontario Tech University, Canada
}

(Communicated to MIIR on 19 October 2021)

Study Group: FM-IPSW 2008, Aug 11-15, 2008, Toronto, Canada

Communicated by: Chris Breward

Industrial Partner: Northern Heavy Industries Group Co., Ltd.

Presenter: Zongxi Cai

Team Members: C. Sean Bohun, Ontario Tech University; Hui-Hui Dai, City University of Hong Kong; Justin Findlay Ontario Tech University; Matt Hennessy Ontario Tech University; Peter Howell, University of Oxford; Huaxiong Huang, York University; Greg Lewis Ontario Tech University; Roderick Melnik, Wilfrid Laurier University; Richard Moore, New Jersey Institute of Technology; Robert Miura, New Jersey Institute of Technology; John Ockendon, University of Oxford; Yonji Tan, Fudan University; Burt Tilley, Olin College of Engineering; Jonathan Wylie, City University of Hong Kong

Industrial Sector: Construction

Key Words: Tunnelling, Soil mechanics, Stability

MSC2020 Codes: 74

\section{Summary}

The force imparted on the soil by a tunnel boring machine can lead to catastrophic tunnel collapse. To help predict this behaviour the machine is modelled as point source in an elastic/plastic idealized soil with no cohesion. The model predicts a wedge shaped region of plasticity extending from the machine. Within this region of plasticity a reduced hyperbolic flow problem determines the dynamics of the soil.

$\dagger$ Corresponding Author: sean. bohun@ontariotechu.ca 


\title{
Interfacial Behaviour in Tunnel Engineering
}

Problem Presenter: Zongxi Cai (Northern Heavy Industries Group Co., Ltd.)

Academic Participants: C. Sean Bohun (UOIT), Hui-Hui Dai (City University of Hong Kong), Justin Findlay (UOIT), Matt Hennessy (UOIT), Peter Howell (University of Oxford), Huaxiong Huang (York University), Greg Lewis (UOIT), Roderick Melnik (Wilfrid Laurier University), Richard Moore (New Jersey Institute of Technology), Robert Miura (New Jersey Institute of Technology), John Ockendon (University of Oxford), Yonji Tan (Fudan University), Burt Tilley (Olin College of Engineering), Jonathan Wylie (City University of Hong Kong)

Report prepared by: C. Sean Bohun ${ }^{1}$

\begin{abstract}
The stress induced in an underground tunnel due to the applied stress of a tunnel boring machine (TBM) is studied to understand the mechanisms which lead to tunnel collapse. An investigation of the classical plane strain gravitational problem in a purely elastic medium shows that any fractures due to gravitational effects alone would occur at the base of the tunnel rather than the apex. Ignoring gravity we then explore an elastic/plastic solution in an idealized soil with no cohesion. To simulate the action of the TBM a point source is imparted on the medium extending in the horizontal direction. This model decouples into an elastostatic problem that predicts a wedge shaped region of plasticity extending from the point source and a hyperbolic flow problem in the plasticized region. Using a similarity solution, it is shown that the behaviour of the medium is very sensitive to the parameters that characterize the soil.
\end{abstract}

\section{Introduction}

Based in Shenyang in the Liaoning Province of China, Northern Heavy Industries Group Co., Ltd. has produced tunnel boring machines (TBMs) since 2005. Some of the advantages of using a TBM compared to other methods of excavation include but are not limited to: an increased rate of advance, a guarantee of the construction times and costs, a higher quality of tunnel, and improved safety conditions.

\footnotetext{
${ }^{1}$ sean.bohun@uoit.ca
} 
For this workshop a number of challenges concerning the operation of the TBM were presented to the group. With respect to the actual cutter head, both the loadings acting on a single cutter and the distribution of the interfacial force across the cutter head were of interest. Of primary interest, was the stability of the interface during drilling and through this the prevention of tunnel collapse. Both the determination of an appropriate constitutive model for the soil the TBM is passing through and the mechanism of any generated instability were specific questions identified by the industrial representative.

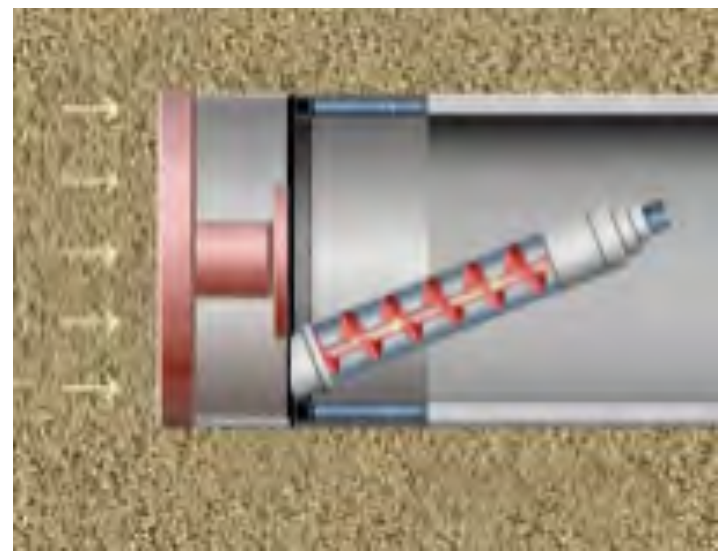

Figure 1 Front assembly of a TBM illustrating the excavation face, the pressurized space and the mechanism for the controlled removal of debris.

Figure 1 shows the operation of a tunnel boring machine. Typically the machine operates between 4 and $80 \mathrm{~m}$ below the surface and extends approximately $400 \mathrm{~m}$ in length and $18 \mathrm{~m}$ in diameter. Progress of the machine (the mucking speed) is quite slow at about $500 \mathrm{~m} /$ month and to stabilize the excavation face, a pressure is applied and removal of the excavated material is controlled as shown by the screw gear in the figure. The objective for the control is to ensure that any surface displacements are between 1 and $3 \mathrm{~cm}$.

The main concern of the Northern Heavy Industries is the prevention of tunnel collapse. This can occur if the applied pressure is too high or the mucking speed is too fast. Figure 2 shows the results of a collapse in a densely populated region. Note that the span of the collapse is quite large. Practical evidence has shown that any collapse typically occurs in advance of the excavation face. There seems to be two distinct scenarios that can lead to collapse. A) If one is progressing through soil then an instability in the excavation face can lead to a collapse of the ground in the immediate neighbourhood of the TBM. Alternatively, B) if the progress is through rock then the induced shock can damage the machine and cause a collapse as it propagates through the soil.

The main goal to be addressed in this report were twofold. First, to determine the best constitutive model for the analysis of the interfacial behaviour. This is actually quite difficult because of the plethora of models available in the literature and it is a bit of a daunting task to find the best model for any particular situation and material being excavated by the TBM. The second goal is to determine the most important mechanisms that lead to instability of the excavation face and some idea of where it might propagate. In addition to these two goals, there were a number of specific goals that we were not able to address namely: i) how to prevent ground surface collapse ii) how to prevent surface uplift 


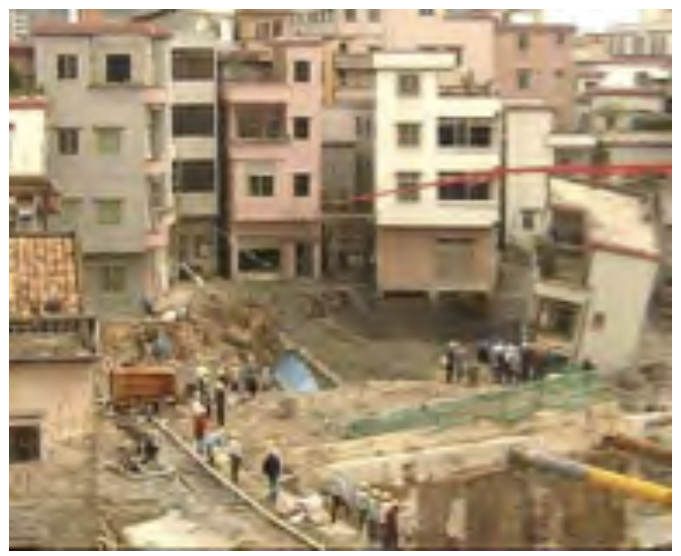

Figure 2 Results of a tunnel collapse in a residential area showing the possibly very large extent.

iii) determination of loadings on a single cutter and iv) the distribution of the forces on the cutter head.

\section{The Effect of Gravity}

The TBM works by shearing away soil and rock and continually moving forward into the newly created space. To keep the soil matrix in place while it is being destabilized, the machine applies a pressure on the order of $2 \mathrm{MPa}$ to the cutting face. As shards of material loosen from the main body they are churned up to create a region of plasticity in front of the machine and it is the behaviour of this region that determines the reaction of the soil to the drilling. Moving away from the cutting head, one expects that the soil would move from this plastic state to a region of relative elasticity. Depending upon the the material being drilled, the size of this plastic region can vary in thickness. There are a number of fundamental processes involved in the creation of a tunnel and understanding the balance between them is the key to preventing tunnel collapse during its creation. These processes are i) the loading pressure that moves the TBM forward into the newly created region; ii) the shear stress applied to the soil matrix used to grind up the material and remove it; and iii) the hydrostatic pressure on the soil due to the soil surrounding the tunnel. This first section looks at the gravitational effects.

We are primarily interested in determining the extent of the region of plasticity from the drill and how the soil moves within it as the drill moves through the soil. Due to the complexity of this problem we will ignore the effect of gravity in the sequel, but it is informative to see it's effect by first reviewing a classical [3] problem. That is, the determination of the stress in the elastic medium surrounding a tunnel of radius $a$ at a depth of $H$.

In the plane strain case the displacement $\mathbf{u}=(u(r, \theta), v(r, \theta), 0)$, and one is led to solve the momentum balance system

$$
\begin{array}{ll}
\frac{1}{r} \frac{\partial}{\partial r}\left(r \sigma_{r r}\right)+\frac{1}{r} \frac{\partial}{\partial \theta} \sigma_{r \theta}-\frac{1}{r} \sigma_{\theta \theta}-\rho g \sin \theta=0, & \sigma_{r r}(a)=0, \\
\frac{1}{r} \frac{\partial}{\partial r}\left(r \sigma_{r \theta}\right)+\frac{1}{r} \frac{\partial}{\partial \theta} \sigma_{\theta \theta}+\frac{1}{r} \sigma_{r \theta}-\rho g \cos \theta=0, & \sigma_{r \theta}(a)=0,
\end{array}
$$


where we have imposed a zero-traction condition on the tunnel wall. Note that in this plane strain limit $\sigma_{z z}=\nu\left(\sigma_{r r}+\sigma_{\theta \theta}\right)$. Finding a solution is more complicated in a multiplyconnected domain, but can be resolved by constructing a modified Airy stress function and considering the case $a \ll H$ so that in the far-field limit $(r \rightarrow \infty)$ one obtains the hydrostatic solution $\sigma_{r r}=\sigma_{\theta \theta}=\rho g(r \sin \theta-H), \sigma_{r \theta}=0$. The resulting components of the stress tensor are [2]

$$
\begin{aligned}
\sigma_{r r} & =\rho g\left(1-\frac{a^{2}}{r^{2}}\right)\left(-H+\left(1+\frac{(1-2 \nu)}{4(1-\nu)} \frac{a^{2}}{r^{2}}\right) r \sin \theta\right) \\
\sigma_{r \theta} & =-\rho g a^{2} \frac{(1-2 \nu)}{4(1-\nu)}\left(1-\frac{a^{2}}{r^{2}}\right) \frac{\cos \theta}{r} \\
\sigma_{\theta \theta} & =\rho g\left(1+\frac{a^{2}}{r^{2}}\right)\left(-H+\frac{(1-2 \nu)}{4(1-\nu)} \frac{a^{2} \sin \theta}{r}\right)+\rho g r \sin \theta \\
\sigma_{z z} & =2 \nu \rho g\left(-H+\left(1+\frac{1}{4(1-\nu)} \frac{a^{2}}{r^{2}}\right) r \sin \theta\right)
\end{aligned}
$$

These components are shown in Figure 3 along with the maximum shear stress given by

$$
S=\max _{i, j} \frac{1}{2}\left|\sigma_{i}-\sigma_{j}\right|
$$

where the $\sigma_{i}$ represent the eigenvalues of the stress tensor. The so-called Tresca criterion proposes that the material will yield and become plastic once $S$ exceed some critical value $\sigma_{Y}$. What is clear from the form of the solution is that by treating the material around the tunnel as an elastic solid, any plastic region in a neighbourhood of the tunnel extends into the earth below the tunnel rather than upwards towards the surface. It is this upwards propagation of a plasticized region of earth that is suspected to lead to tunnel collapse.

Within the elastic region the Navier equations are supplemented with the constitutive relationship the connects the stresses and strains in the material and an appropriate set of boundary conditions. The material remains elastic provided $S<\sigma_{Y}$ as discussed above. Once the material becomes plastic then the simplest model is to simply replace the constitutive relationship with the possibly nonlinear condition $S=\sigma_{Y}$. The free boundary between the elastic and plastic regions being determined by imposing a continuity condition. See [2] for an in depth discussion of these aspects.

\section{Yield Criterion for Soils}

The mechanical properties of soil vary greatly depending upon the nature of a given sample. At one end of the spectrum is dry sand, with no cohesive bond between the individual particles, to that of plastic clay with strong bonds holding the clay particles together. To develop an expression for the yield criterion, we consider a two-dimensional sample of dry granular material that is in static equilibrium as shown in Figure 4.

Acting on this surface are a normal traction $N$ and frictional stress $F$. These are obtained from the stress tensor by taking the corresponding component of the stress in the 


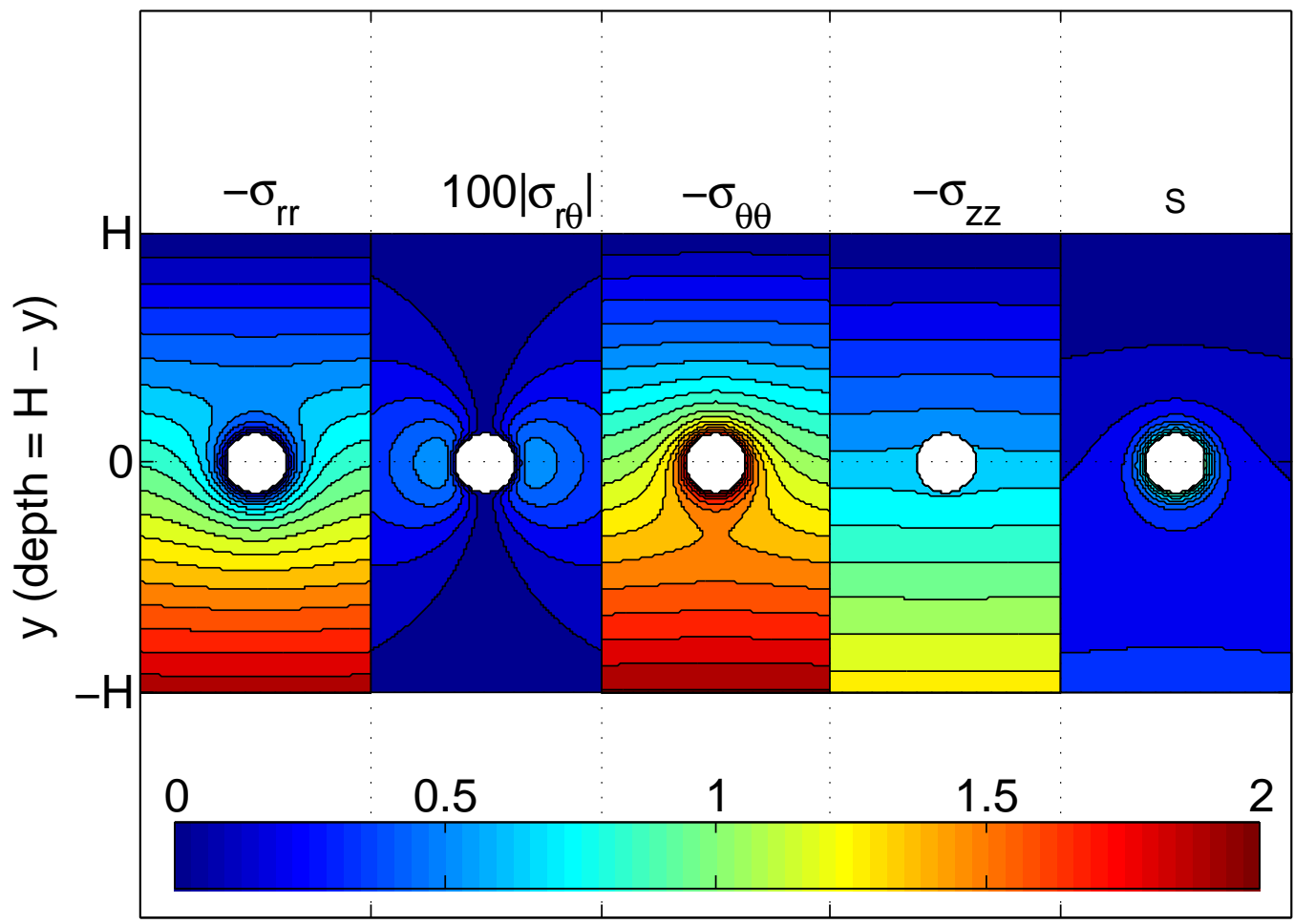

Figure 3 The elements of the stress tensor in units of $\rho g H$ due to a long tunnel at a depth of $H$ of radius $a \ll H$ in a purely elastic medium. In simulation $\nu=1 / 3, H=20 \mathrm{~m}$ and $a=2.5 \mathrm{~m}$. To the far right is a plot of the maximum shear stress $S$ and illustrates that any plastic region extends into the below the tunnel rather than towards the surface.

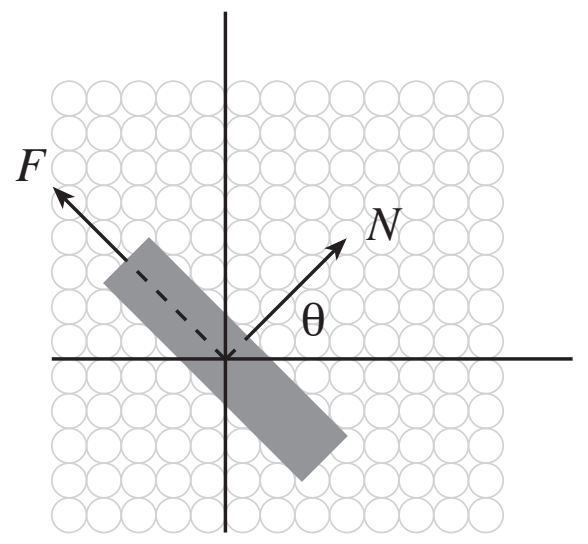

Figure 4 An interior surface element of a granular material in static equilibrium at an angle $\theta . N$ is the normal force of the material above the element acting on the material below while $F$ is the tangential frictional force between the granules.

plane of the surface element so that

$$
\begin{aligned}
N & =\left(\begin{array}{ll}
\cos \theta & \sin \theta
\end{array}\right)\left(\begin{array}{ll}
\sigma_{x x} & \sigma_{x y} \\
\sigma_{x y} & \sigma_{y y}
\end{array}\right)\left(\begin{array}{c}
\cos \theta \\
\sin \theta
\end{array}\right) \\
& =\frac{1}{2}\left(\sigma_{x x}+\sigma_{y y}\right)+\frac{1}{2}\left(\sigma_{x x}-\sigma_{y y}\right) \cos 2 \theta+\sigma_{x y} \sin 2 \theta, \\
F & =\left(\begin{array}{ll}
-\sin \theta & \cos \theta
\end{array}\right)\left(\begin{array}{ll}
\sigma_{x x} & \sigma_{x y} \\
\sigma_{x y} & \sigma_{y y}
\end{array}\right)\left(\begin{array}{c}
\cos \theta \\
\sin \theta
\end{array}\right) \\
& =\frac{1}{2}\left(\sigma_{y y}-\sigma_{x x}\right) \sin 2 \theta+\sigma_{x y} \cos 2 \theta .
\end{aligned}
$$


Combining (3.1a) and (3.1b) yields the identity

$$
F^{2}+\left(N-\frac{1}{2}\left(\sigma_{x x}+\sigma_{y y}\right)\right)^{2}=\frac{1}{4}\left(\sigma_{x x}-\sigma_{y y}\right)^{2}+\sigma_{x y}^{2}
$$

which describes a circle in the $(F, N)$ plane known as the Mohr circle. ${ }^{2}$

Since the material cannot withstand any tensile stress, we must have $N<0$ and by Coulomb's law the material will be held in place by friction provided that $|F|<\mu_{f}|N|$ where $\mu_{f}$ is the coefficient of friction. Defining the angle of friction $\phi$ so that $\mu_{f}=\tan \phi$ gives the expression $|F|<|N| \tan \phi$. For material that is flowing there must be a single value of $\theta$ such that $|F|=|N| \tan \phi$ so that in the $(F, N)$ plane, this line must necessarily be tangent to the Mohr circle. Using (3.2) one is lead to the Mohr-Coulomb yield criterion

$$
f\left(\sigma_{i j}\right)=\left(\frac{1}{4}\left(\sigma_{x x}+\sigma_{y y}\right)^{2}-\left(\sigma_{x x} \sigma_{y y}-\sigma_{x y}^{2}\right)\right)^{1 / 2}+\frac{1}{2}\left(\sigma_{x x}+\sigma_{y y}\right) \sin \phi \leq 0
$$

with equality when the material is flowing. Any cohesion due to soil moisture, electrostatic charges between granules, or perhaps another mechanism can be modelled by mapping $N \mapsto N+C$ where $C$ is a positive cohesion force. In this case (3.3) becomes

$$
\left(\frac{1}{4}\left(\sigma_{x x}+\sigma_{y y}\right)^{2}-\left(\sigma_{x x} \sigma_{y y}-\sigma_{x y}^{2}\right)\right)^{1 / 2}+\frac{1}{2}\left(\sigma_{x x}+\sigma_{y y}\right) \sin \phi \leq C \sin \phi .
$$

The direction angle $\theta$ associated with the yield criterion defines a slip surface along which the flow is expected to take place. More details on the slip line analysis can be found in [4] and an in depth treatment of the theories of soil mechanics is available in [6].

\section{Elastic/Plastic Transition}

Plastic materials are characterized by a stress/strain curve that is elastic up to some material dependent critical value. Beyond this value the material flows resulting in permanent strain. To illustrate the elastic/plastic transition in an idealized situation, we consider a situation described in [2] of a tunnel of radius $a$ in an infinite domain while ignoring gravity. At $r=a$ it is supposed that there is a pressure $P_{0}$ to simulate bracing, and a uniform pressure $P_{\infty}$ as one moves away in any direction. Assuming a displacement of the form $\mathbf{u}=(u(r), 0,0)$ only the nontrivial strains are $\epsilon_{r r}=u^{\prime}$ and $\epsilon_{\theta \theta}=u / r$. This leaves only the single Navier equation

$$
\frac{\mathrm{d}}{\mathrm{d} r} \sigma_{r r}+\frac{1}{r}\left(\sigma_{r r}-\sigma_{\theta \theta}\right)=0
$$

with boundary conditions

$$
\sigma_{r r}(a)=-P_{0}, \quad \quad \lim _{r \rightarrow \infty} \sigma_{r r}(r)=\lim _{r \rightarrow \infty} \sigma_{\theta \theta}(r)=-P_{\infty} .
$$

In the elastic region one has the constitutive laws

$$
\sigma_{r r}=(\lambda+2 \mu) \frac{\mathrm{d} u}{\mathrm{~d} r}+\lambda \frac{u}{r}, \quad \sigma_{\theta \theta}=(\lambda+2 \mu) \frac{u}{r}+\lambda \frac{\mathrm{d} u}{\mathrm{~d} r},
$$

and solving for the displacement $u(r)$ determines the components of the stress tensor

$$
\sigma_{r r}=-P_{\infty}+\left(P_{\infty}-P_{0}\right) \frac{a^{2}}{r^{2}}, \quad \sigma_{\theta \theta}=-P_{\infty}-\left(P_{\infty}-P_{0}\right) \frac{a^{2}}{r^{2}}, \quad \sigma_{z z}=-2 \nu P_{\infty}
$$

\footnotetext{
${ }^{2}$ In three dimensions a similar condition is satisfied but only with respect to the principal axes so that the stress tensor takes the form $\sigma=\operatorname{diag}\left(\sigma_{1}, \sigma_{2}, \sigma_{3}\right)$.
} 
In this case the yield criterion (3.3) is

$$
f\left(\sigma_{i j}\right)=\left(\frac{1}{4}\left(\sigma_{r r}+\sigma_{\theta \theta}\right)^{2}-\left(\sigma_{r r} \sigma_{\theta \theta}-\sigma_{r \theta}^{2}\right)\right)^{1 / 2}+\frac{1}{2}\left(\sigma_{r r}+\sigma_{\theta \theta}\right) \sin \phi=0
$$

which simplifies to $\sigma_{\theta \theta}=k \sigma_{r r}$ where the triaxial stress factor $k$ satisfies

$$
k=\frac{1+\sin \phi}{1-\sin \phi} .
$$

Looking at the ratio $\sigma_{\theta \theta} / \sigma_{r r}$ at $r=a$ we see that the material remains elastic provided that the bracing pressure $P_{0}$ does not fall below the critical value

$$
P_{\text {crit }}=\frac{2}{k+1} P_{\infty}
$$

Below this critical pressure a plastic region extends from the tunnel wall at $r=a$ to a value of $r=s$ within which the stress components satisfy

$$
\frac{\mathrm{d}}{\mathrm{d} r} \sigma_{r r}+\frac{1}{r}\left(\sigma_{r r}-\sigma_{\theta \theta}\right)=0, \quad \sigma_{\theta \theta}=k \sigma_{r r}, \quad \sigma_{r r}(a)=-P_{0}>-P_{\text {crit }}
$$

with solution

$$
\sigma_{r r}=-P_{0}\left(\frac{r}{a}\right)^{k-1}, \quad \sigma_{\theta \theta}=-k P_{0}\left(\frac{r}{a}\right)^{k-1}, \quad \sigma_{z z}=-2 \nu P_{\infty} \frac{P_{0}}{P_{\text {crit }}}\left(\frac{r}{a}\right)^{k-1} .
$$

Taking (4.4), (4.7) and (4.9) together gives the composite elastic-plastic solution

$$
\begin{gathered}
\sigma_{r r}=\left\{\begin{array}{ccc}
-P_{0}\left(\frac{r}{a}\right)^{k-1}, & a \leq r \leq s, \quad P_{0}<P_{\text {crit }}, \\
-P_{\infty}+P_{\infty}\left(\frac{k-1}{k+1}\right) \frac{s^{2}}{r^{2}}, & r>s, & P_{0}<P_{\text {crit }}, \\
-P_{\infty}+\left(P_{\infty}-P_{0}\right) \frac{a^{2}}{r^{2}}, & r \geq a, & P_{0} \geq P_{\text {crit }},
\end{array}\right. \\
\sigma_{\theta \theta}=\left\{\begin{array}{ccc}
-k P_{0}\left(\frac{r}{a}\right)^{k-1}, & a \leq r \leq s, \quad P_{0}<P_{\text {crit }}, \\
-P_{\infty}-P_{\infty}\left(\frac{k-1}{k+1}\right) \frac{s^{2}}{r^{2}}, & r>s & P_{0}<P_{\text {crit }}, \\
-P_{\infty}-\left(P_{\infty}-P_{0}\right) \frac{a^{2}}{r^{2}}, & r \geq a, & P_{0} \geq P_{\text {crit }} .
\end{array}\right.
\end{gathered}
$$

To find the location $s$ we impose that $\sigma_{r r}$ is continuous as $r=s$ for $P_{0}<P_{\text {crit }}$ which gives the expression for

$$
s=a\left(\frac{P_{\text {crit }}}{P_{0}}\right)^{1 /(k-1)} .
$$

Figure 5 illustrates the radial behaviour of the stress for a friction angle of $\phi=30$ degrees and and a tunnel wall pressure of $P_{0}=P_{\text {crit }} / 2$. We close this section by noting that there is a significant amount of classical work done modelling tunnels in granular rock and we refer the reader to [2] and the references therein for further detailed analysis. 


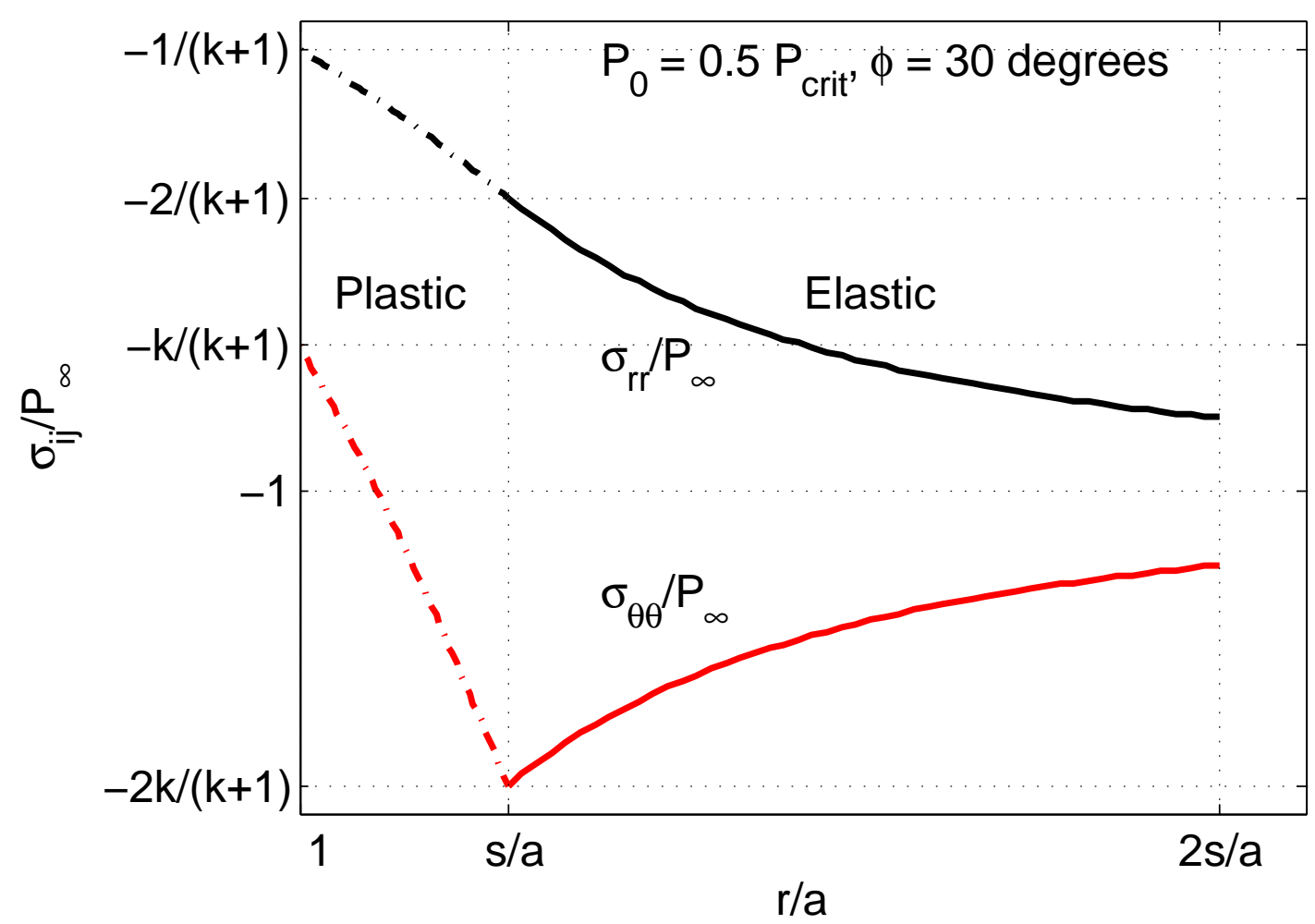

Figure 5 The radial and hoop stress in the material surrounding a tunnel of radius $a$ when the pressure at the tunnel wall is $P_{0}=P_{\text {crit }} / 2$. In this example the angle if friction is taken to be 30 degrees so that the triaxial stress factor $k=3$ and $P_{\text {crit }}=P_{\infty} / 2$.

\section{The Model}

What we would like to understand with respect to the operation of the TBM is the dynamics of any plasticized region extending from the tunnel to perhaps the surface. The equations of plasticity must include a form of momentum balance, a yield criterion and some form of associated flow rule. The flow $\mathbf{v}$ associated with a displacement $\mathbf{u}$ is given by

$$
\mathbf{v}=\frac{\partial \mathbf{u}}{\partial t}+(\mathbf{v} \cdot \nabla) \mathbf{u}
$$

or solving for $\mathbf{v}$ explicitly,

$$
\mathbf{v}=\left(I-\nabla \mathbf{u}^{\mathrm{T}}\right)^{-1} \frac{\partial \mathbf{u}}{\partial t} \simeq \frac{\partial \mathbf{u}}{\partial t}
$$

for small displacements. The Cauchy equation is similarly modified to

$$
\nabla \cdot \sigma+\rho \mathbf{g}=\rho\left(\frac{\partial \mathbf{v}}{\partial t}+(\mathbf{v} \cdot \nabla) \mathbf{v}\right) \simeq 0
$$

by neglecting any accelerations in the flow. A flow rule associated with the yield criterion $f\left(\sigma_{i j}\right)=0$ can be arrived at by considering the heat dissipated during a deformation, and maximizing the rate at which heat is dissipated. As shown in [2], the heat dissipated during 
a deformation is given by $\Phi=D_{i j} \sigma_{i j}$ where

$$
D_{i j}=\frac{1}{2}\left(\frac{\partial v_{i}}{\partial x_{j}}+\frac{\partial v_{j}}{x_{i}}\right)
$$

is the rate of strain tensor. For small displacements $D_{i j} \simeq \partial e_{i j} / \partial t$, the time rate of change of the linear strain tensor. To maximize $\Phi$, subject to the constraint $f\left(\sigma_{i j}\right)=0$, we find

$$
\frac{\partial e_{i j}}{\partial t} \simeq D_{i j}=\lambda \frac{\partial f}{\partial \sigma_{i j}}
$$

for some value $\lambda \in \mathbb{R}$.

Although gravity plays a role in the stress field as shown earlier in this report, compared to the magnitude of the forces imparted at the drill head it can be effectively ignored. We are also primarily interested in the behaviour of shallow tunnels where gravitational effects are suppressed. Combining all these approximations gives a system of ten equations

$$
\frac{\partial \sigma_{i j}}{\partial x_{j}}=0, \quad \frac{1}{2}\left(\frac{\partial v_{i}}{\partial x_{j}}+\frac{\partial v_{j}}{x_{i}}\right)=\lambda \frac{\partial f}{\partial \sigma_{i j}}, \quad f\left(\sigma_{i j}\right)=0
$$

in ten unknowns; ( $\operatorname{six} \sigma_{i j}$, three $v_{i}$ and one $\lambda$ ).

5.1 The elastostatic problem. Starting with the simplest configuration we consider the plane strain case in the $x y$-plane with a displacement of $\mathbf{u}=(u(x, y), v(x, y), 0)$ with a point force in the positive $x$ direction. Considering only the static case, we are left with the system

$$
\frac{\partial \sigma_{x x}}{\partial x}+\frac{\partial \sigma_{x y}}{\partial y}=-F \delta(x) \delta(y), \quad \frac{\partial \sigma_{x y}}{\partial x}+\frac{\partial \sigma_{y y}}{\partial y}=0 .
$$

Solving (5.7) using a Love function [2] results in a stress tensor with components ${ }^{3}$

$$
\begin{aligned}
\sigma_{x x} & =\frac{F}{4 \pi(1-\nu) r^{4}}\left(-(3-2 \nu) x^{3}-(1-2 \nu) x y^{2}\right), \\
\sigma_{x y} & =\frac{F}{4 \pi(1-\nu) r^{4}}\left(-(3-2 \nu) x^{2} y-(1-2 \nu) y^{3}\right), \\
\sigma_{y y} & =\frac{F}{4 \pi(1-\nu) r^{4}}\left((1-2 \nu) x^{3}-(1+2 \nu) x y^{2}\right) .
\end{aligned}
$$

The eigenvalues of the stress tensor are

$$
\lambda_{ \pm}=\frac{F}{4 \pi(1-\nu) r^{2}}\left(x \pm\left(4(1-\nu)^{2} x^{2}+(1-2 \nu)^{2} y^{2}\right)^{1 / 2}\right)
$$

and associated with this is a maximal shear stress of

$$
F_{\max }=\frac{1}{2}\left|\lambda_{+}-\lambda_{-}\right|=\frac{F}{4 \pi(1-\nu) r^{2}}\left(4(1-\nu)^{2} x^{2}+(1-2 \nu)^{2} y^{2}\right)^{1 / 2}
$$

and a maximal normal traction of

$$
N_{\max }=\max \lambda_{ \pm}=\frac{F}{4 \pi(1-\nu) r^{2}} \max \left(x \pm\left(4(1-\nu)^{2} x^{2}+(1-2 \nu)^{2} y^{2}\right)^{1 / 2}\right) .
$$

\footnotetext{
${ }^{3}$ As with many elastostatic problems, the phrase "all you need is Love" comes to mind.
} 
Contour plots these maximal stresses are illustrated in Figure 6. Applying this stress field to the yield criterion gives the condition

$$
\left(4(1-\nu)^{2} x^{2}+(1-2 \nu)^{2} y^{2}\right)^{1 / 2}-4 x \sin \phi=0
$$

for the boundary between the elastic and plasticized region. Note that we only have a nontrivial region if $|\sin \phi|>1-\nu$ or about 42 degrees for $\nu=1 / 3$. The nontrivial region forms a wedge extending with an opening angle of $2 \beta$ where

$$
\tan ^{2} \beta=4 \frac{\sin ^{2} \phi-(1-\nu)^{2}}{(1-2 \nu)^{2}} .
$$

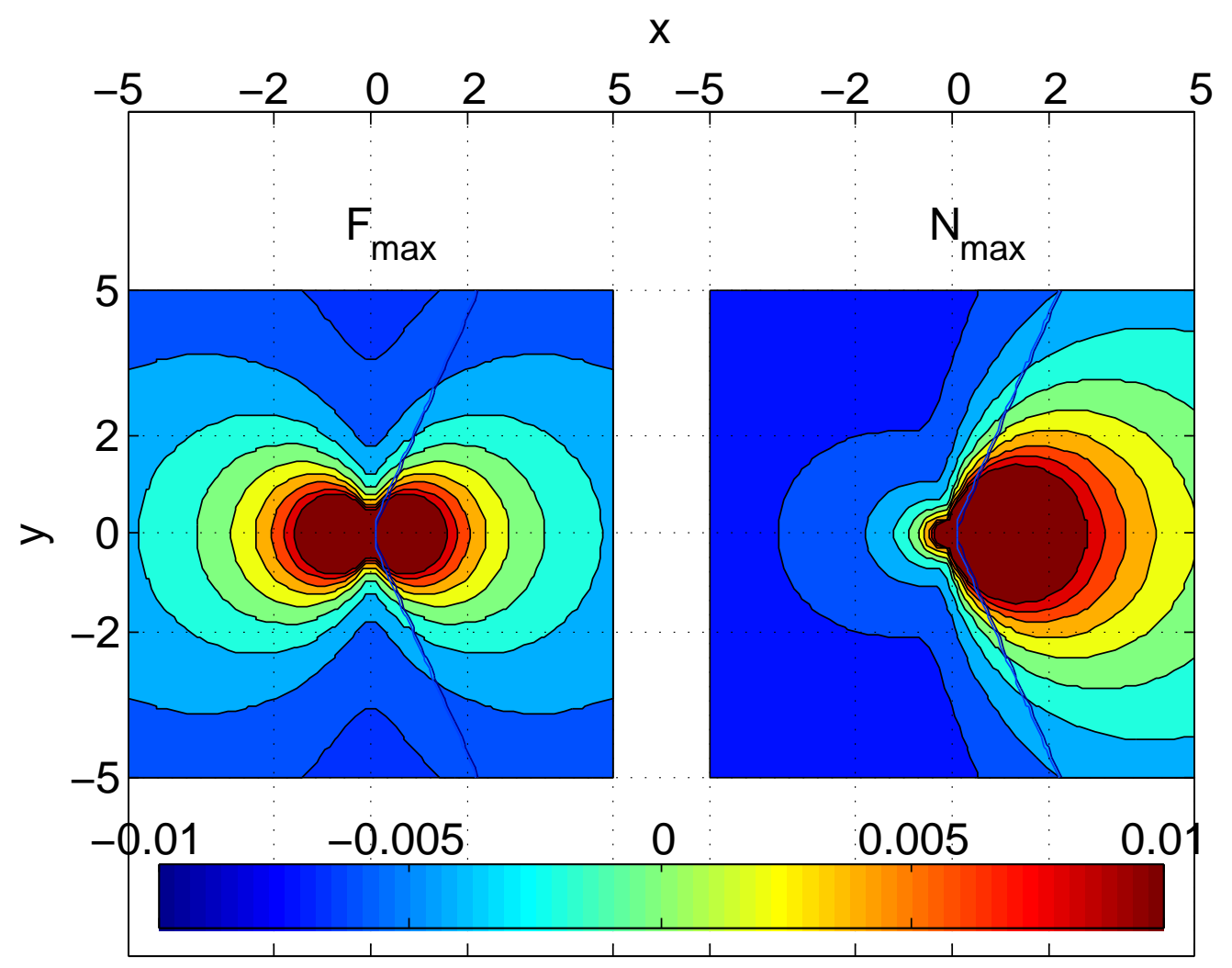

Figure 6 The maximum shear stress $F_{\max }$ and the maximal normal traction $N_{\max }$ for the point source of strength $F=1$ and $\nu=1 / 3$. The blue lines illustrates the edge of the plasticized region where $f\left(\sigma_{i j}\right)=0$ for $\phi=50$ degrees.

For $\delta=50$ degrees this is an opening angle of $2 \beta=132$ degrees. This region is also depicted in the figure. Figure 7 shows the sensitivity of $\beta$ with the friction angle $\phi$. Small variations in $\delta$ generate large changes in $\beta$ which indicates that subtle changes in the soil frictional qualities can lead to very large changes in the domain of the plasticized region.

5.2 The flow problem. Turning now to the corresponding flow problem, we note that if the stress problem is formulated in terms of an Airy stress function, then the resulting PDE is hyperbolic. As a result, disturbances are expected to propagate through the soil. This mechanism is thought to be a key factor in predicting whether or not there is a tunnel collapse. This line of reasoning was motivated by the fact that the two dimensional 


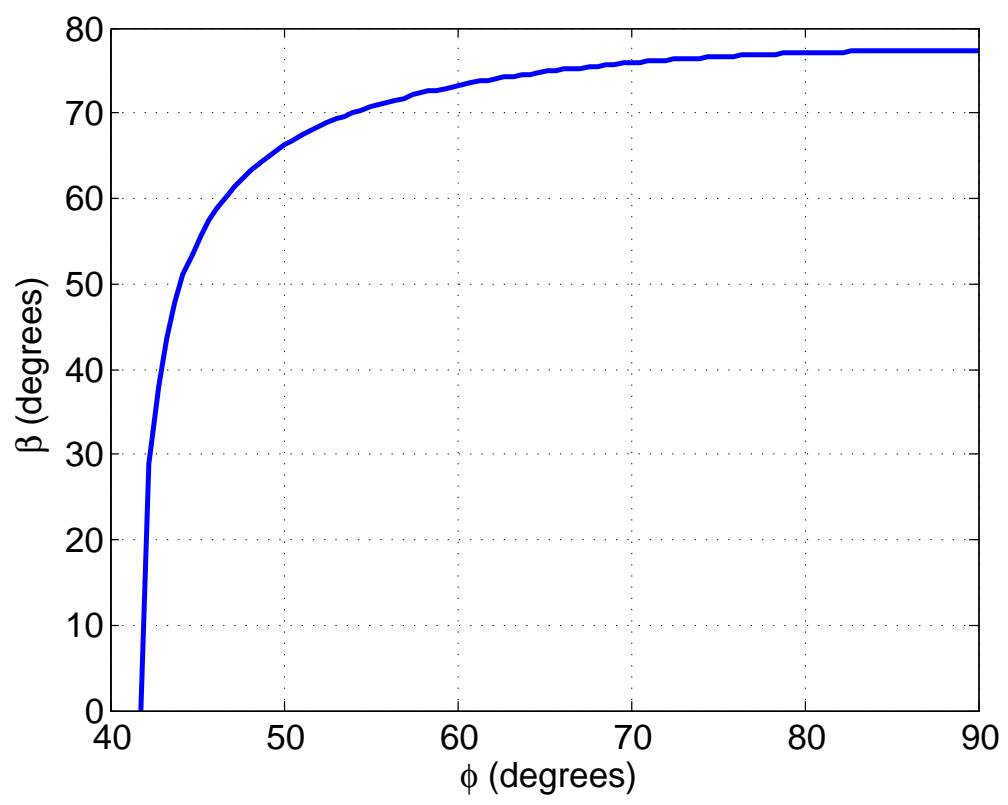

Figure 7 Dependence of one-half the opening angle, $\beta$, with respect to the friction angle $\phi$ according to (5.11) for $\nu=1 / 3$.

problem can be written as a hyperbolic system in the variables $p=-\left(\sigma_{x x}+\sigma_{y y}\right) / 2$ and $\tan 2 \psi=2 \sigma_{x y} /\left(\sigma_{x x}-\sigma_{y y}\right)$ with corresponding characteristics

$$
\lambda_{ \pm}=\tan \left(\psi+\frac{\pi}{2} \pm\left(\frac{\pi}{4}+\frac{\phi}{2}\right)\right)
$$

and Riemann invariants

$$
R^{ \pm}=2 \psi \pm \cot \phi \log p .
$$

See $[1,5]$ for a direction application of this to the flow of granular material in a grain hopper.

Returning to the (5.6) we have the momentum balance,

$$
\begin{aligned}
& \frac{\partial \sigma_{r r}}{\partial r}+\frac{1}{r} \frac{\partial \sigma_{r \theta}}{\partial \theta}+\frac{\sigma_{r r}-\sigma_{\theta \theta}}{r}=0, \\
& \frac{\partial \sigma_{r \theta}}{\partial r}+\frac{1}{r} \frac{\partial \sigma_{\theta \theta}}{\partial \theta}+\frac{2 \sigma_{r \theta}}{r}=0,
\end{aligned}
$$

the equation of state for the plasticized region (4.5) and three expressions defining the flow $\mathbf{v}=\left(v_{r}, v_{\theta}\right)$

$$
\frac{\partial v_{r}}{\partial r}=\lambda \frac{\partial f}{\partial \sigma_{r r}}, \quad \frac{1}{r} \frac{\partial v_{r}}{\partial \theta}+\frac{\partial v_{\theta}}{\partial r}-\frac{v_{\theta}}{r}=2 \lambda \frac{\partial f}{\partial \sigma_{r \theta}}, \quad \frac{1}{r}\left(\frac{\partial v_{\theta}}{\partial \theta}+v_{r}\right)=\lambda \frac{\partial f}{\partial \sigma_{r r}} .
$$

The elastic solution (5.8) is consistent with an isotropic elastic medium so it is not applicable for this plasticized situation. However, if we suppose that the tunnel is deep enough so that there are no boundary effects, then we can search for a similarity solution. Motivated by the solution (5.8) we choose ${ }^{4} \sigma_{\theta \theta}=A \cos \theta / r$ and the momentum equations are satisfied

\footnotetext{
${ }^{4} \sigma_{\theta \theta}=\sigma_{y y} \cos ^{2} \theta+\sigma_{x x} \sin ^{2} \theta+2 \sigma_{x y} \cos \theta \sin \theta$.
} 
provided

$$
\sigma_{\theta \theta}=\frac{A \cos \theta}{r}, \quad \sigma_{r \theta}=\frac{A \sin \theta}{r}, \quad \sigma_{r r}=\frac{A g(\theta)}{r}
$$

for any function $g(\theta)$. The form of $g(\theta)$ is chosen to ensure that the stress field is consistent with the yield surface (4.5) and one can verify that

$$
g(\theta)=\left(2 \sec ^{2} \phi-1\right) \cos \theta+\sec ^{2} \phi(2(\cos 2 \theta-\cos 2 \phi))^{1 / 2}
$$

accomplishes this. Using these expressions for the stress a similarity solution for the flow takes the form

$$
\lambda=\gamma(\theta) r^{\alpha-1}, \quad v_{r}=w_{r}(\theta) r^{\alpha}, \quad v_{\theta}=w_{\theta}(\theta) r^{\alpha}
$$

and transforms the flow rule into the nonstandard eigenvalue problem for the plasticized region $-\delta<\theta<\delta$

$$
\frac{\mathrm{d}}{\mathrm{d} \theta}\left(\begin{array}{l}
w_{r} \\
w_{\theta}
\end{array}\right)=\left(\begin{array}{cc}
\frac{2 \alpha f_{r \theta}}{f_{r r}} & -\alpha \\
\frac{\alpha f_{\theta \theta}}{f_{r r}}-1 & 0
\end{array}\right)\left(\begin{array}{l}
w_{r} \\
w_{\theta}
\end{array}\right), \quad\left(\begin{array}{l}
w_{r} \\
w_{\theta}
\end{array}\right)( \pm \delta)=0,
$$

where $f_{i j}=\partial f / \partial \sigma_{i j}$ and the boundary condition at $\theta= \pm \delta$ enforces a no-flow condition where $g$ becomes complex valued.

\section{Results}

The flow problem was solved numerically for a range of friction angles. In particular, $\phi=30,40$ and 50 degrees and displayed in Figure 8. Note that the point source solution is not a similarity solution and some care must be taken when comparing the elastostatic problem with the solution of the flow problem. What is clear is that as $\delta$ is increased, the displacement becomes larger at the source of the disturbance and increasingly localized. Eventually a critical value of $\phi$ is reached and $\alpha$ becomes complex which results in a churning flow where material is forced from the outer edges back towards the drill head. This sets up instabilities in the soil matrix that propagate a significant distance from the head of the drill. It is suspected that this phenomenon could be one responsible for the collapse. It is encouraging that this bifurcation occurs somewhere near $\delta=48$ degrees with simulations using $\nu=1 / 3$ and that the opening angle of the plasticized region increases with $\delta$ comparable with that predicted by the elastostatic point source case.

\section{Conclusions and Future Work}

Working in a simplified geometry the tunnel collapse problem was decoupled into an elastostatic point source stress field and a flow model for the plasticized region that maximizes heat dissipation in the media. This latter model leads to a hyperbolic equation for the flow velocity whose behaviour is highly sensitive to the soil parameters.

What has been made quite clear, by studying these geometrically simplified problems, is that understanding the material properties of the soil prior to tunnelling is a key to preventing collapse. The extent of the plasticized region depends strongly on this behaviour yet saturates quickly in extreme cases. This latter fact lends itself to the possibility of predicting a minimal depth that the TBM would have to operate to ensure no collapse occurs in a given worst case scenario. 

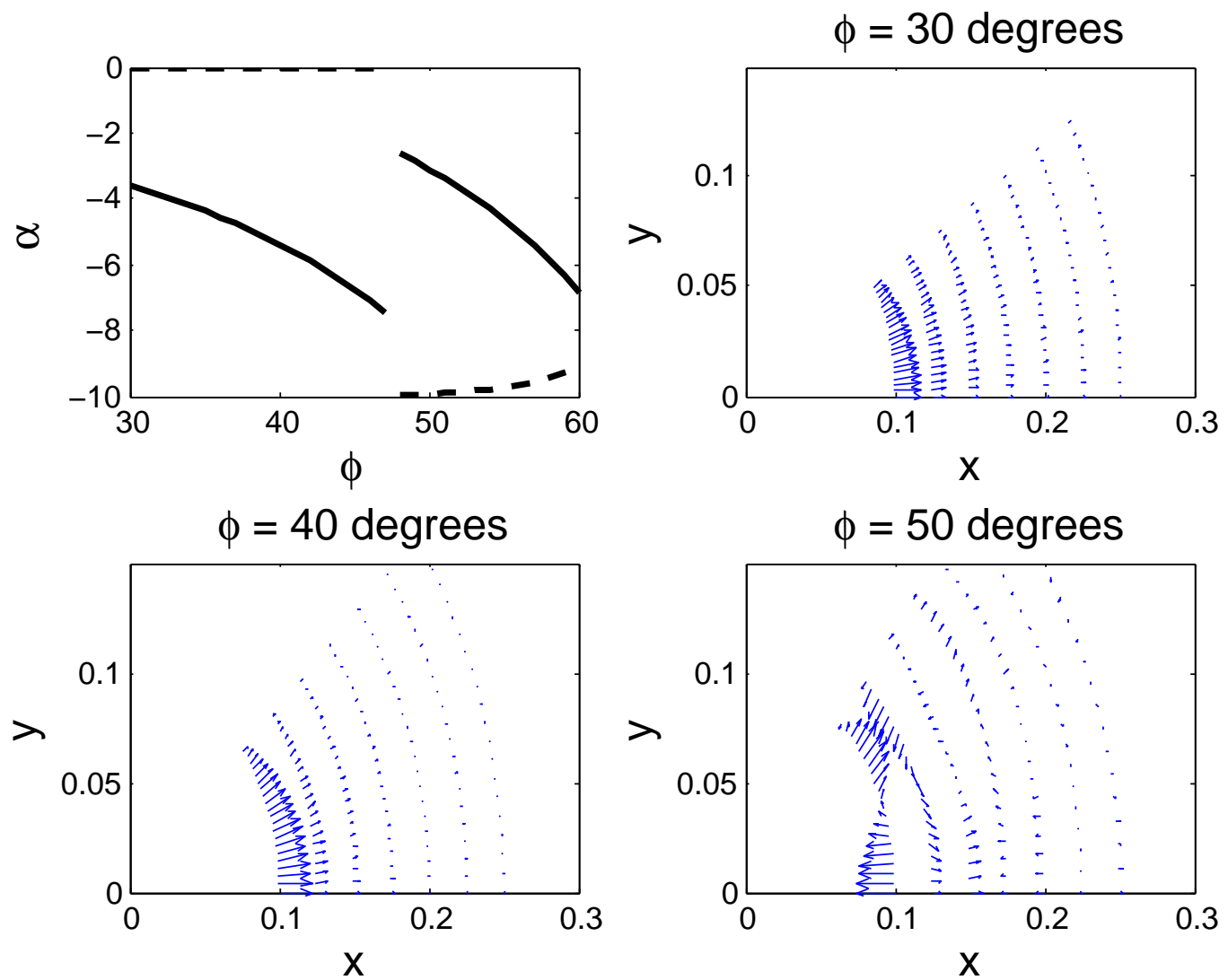

Figure 8 The eigenvalue $\alpha$ as a function of the parameter $\phi$ and some of the associated flow patterns. The solid line denotes the real part of $\alpha, \Re(\alpha)$ and the dashed line corresponds to the imaginary part, $\Im(\alpha)$. Somewhere near $\delta=48$ degrees the value of $\alpha$ becomes complex and the flow begins to churn.

A model taking gravity into effect illustrates that hydrostatic pressure would tend to generate a plasticized region below the tunnel rather than above it. However, gravitational effects can deflect the surface of transition between the elastic and plastic region. For this reason, some future effort should be made to include gravity systematically. Provided it is small compared to the applied forces, this solution could be matched to the far-field behaviour. Related to this, the inclusion of a stress-free boundary condition at the free surface should be included to determine its effect on the instability of the soil matrix. Also of interest is an axisymmetric problem with an axis coincident with the tunnel rather than a polar plane strain situation described.

Other problems that were not explored but which may prove useful include the following: (i) Dealing with the finite extent of the drill head rather than a point source. In this situation the motion of the drilling head should be incorporated as a boundary condition for the flow model. (ii) Treating the situation where the soil properties vary spatially. This is one of the most relevant problems for tunnel collapse yet also remains one of the most challenging to solve. 


\section{Acknowledments}

The author would like to thank Greg Lewis for his infinite patience in waiting for this report. I'm not sure I would have been as lenient with such a long delay. In closing, I would like to remark that in the final weeks of preparation of this material, a TBM in Niagara Falls Ont. completed digging a massive tunnel under the falls itself. The 30 tonne TBM known as "Big Becky" started in 2006 and completed the 10.2 kilometre tunnel, $90 \mathrm{~m}$ to $140 \mathrm{~m}$ in depth, as part of the construction of a hydroelectric infrastructure project in the Niagara region. This tunnel is roughly 150\% larger than the Chunnel linking England and France under the English channel. Completing the report as this announcement was made was prophetic.

\section{References}

[1] Brennan, C. \& Pearce, J. (1978). Granular material flow in two-dimensional hoppers, Journal Applied Mechanics, American Society of Mechanical Engineers, 45, pp. 43-50.

[2] Howell, P., Kozyreff, G. \& Ockendon, J. (2009). Applied Solid Mechanics, Cambridge Texts in Applied Mathematics.

[3] Mindlin, R.D. (1939). Stress distribution around a tunnel. Proceedings of the American Society of Civil Engineers, 65(4), pp. 619-642.

[4] Richards, R. (2001). Principles of Solid Mechanics, CRC Press LLC.

[5] Tayler, A.B. (1986). Mathematical models in applied mechanics, Claringdon Press, Oxford.

[6] Terzaghi, K. \& Peck, R.B. (1948). Soil Mechanics in Engineering Practice, Wiley, New York. 